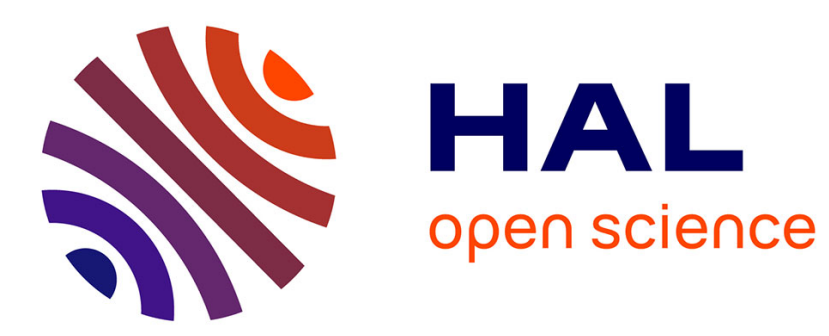

\title{
Une lecture des styles de conjugalité au sein de l'habitat des couples lesbiens
}

Céline Costechareire

\section{To cite this version:}

Céline Costechareire. Une lecture des styles de conjugalité au sein de l'habitat des couples lesbiens. Politiques sociales et familiales, 2011, 103, pp 65-79. halshs-00588731

\section{HAL Id: halshs-00588731 https://shs.hal.science/halshs-00588731}

Submitted on 26 Apr 2011

HAL is a multi-disciplinary open access archive for the deposit and dissemination of scientific research documents, whether they are published or not. The documents may come from teaching and research institutions in France or abroad, or from public or private research centers.
L'archive ouverte pluridisciplinaire HAL, est destinée au dépôt et à la diffusion de documents scientifiques de niveau recherche, publiés ou non, émanant des établissements d'enseignement et de recherche français ou étrangers, des laboratoires publics ou privés. 


\title{
Une lecture des styles de conjugalité au sein de l'habitat des couples lesbiens
}

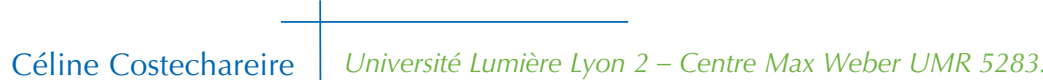 \\ Mots clés : Couples lesbiens - Style de conjugalité - Habitat.
}

En France, les couples lesbiens restent encore mal connus en sociologie de la famille. Ils permettent pourtant d'aborder et d'observer la conjugalité sous un angle nouveau. Comment s'organisent les unions féminines? Diffèrent-elles des unions de même sexe? Par une analyse de la décoration, des différents modes d'appropriation spatiale et des sociabilités qui se profilent au sein de l'habitat, cet article présente la pluralité des modèles conjugaux qui se dessine chez les couples lesbiens. La description des organisations conjugales fait écho à la diversité des styles conjugaux qui s'exprime chez les couples hétérosexuels. On observe, en effet, d'un côté, des couples lesbiens plutôt fusionnels, accordant un privilège marqué au "nous " conjugal et, de l'autre, des couples plus ouverts sur l'extérieur, dont l'organisation révèle la prépondérance de l'individualité de chaque partenaire.

u carrefour de la sociologie de la famille et de Ala sociologie de l'espace, l'étude présentée dans cet article vise à saisir les différents styles conjugaux qui se profilent au sein de l'habitat des couples lesbiens. En France, les unions lesbiennes demeurent encore mal connues. Alors que les études consacrées à l'homoparentalité ont pris un essor considérable durant cette dernière décennie (Descoutures, 2008 et 2010 ; Goldberg et Allen, 2009; Gratton, 2008; Gross, 2005 et 2009; Mailfert, 2005 et 2007), que les unions de même sexe sont abordées sous le prisme de l'homosexualité masculine (Courduriès, 2006 et 2008; Lerch, 2007), seuls quelques travaux traitent de I'homosexualité et de I'homoconjugalité féminine (Chetcuti, 2010 ; Costechareire, 2008 ; Ferzli, 2001). Les recherches menées sur les couples hétérosexuels sont, quant à elles, conséquentes ; elles ont ainsi fortement contribué à poser les jalons théoriques de cette étude des unions féminines.
Les couples lesbiens sont analysés dans cette enquête (1) par le biais du "système de relations » qui s'établit entre I'espace habité et le couple habitant (Allen, 2004). Le couple se construit "à petits pas ». Il s'inscrit dans "un processus lent de formation " au cours duquel se cimentent des habitudes et se répartissent des rôles entre les partenaires (Kaufmann, 2003:60). Il s'édifie dans un espace dont l'acception apparaît double. Cette acception désigne l'espace de la relation entre conjoint-e-s et l'espace physique au sein duquel se tisse quotidiennement la trame de cette relation. La première dimension implique une analyse des échanges et des négociations entre partenaires participant $d^{\prime}$ un style conjugal. La deuxième inclut la notion $d^{\prime}$ " habitat ». Porteur de valeurs affectives et d'enjeux identitaires (Allen, 2004 ; Bonnin, 1990), I'habitat affiche l'empreinte de ses occupants et celle de la conjugalité. II permet I'analyse des relations familiales [Singly (de), 1996], se présente comme une scène des interactions conjugales (Kaufmann, 1991), et cristallise à ce titre les modalités de la relation qui se déroule en son sein. L'habitat participe, en outre, de la construction conjugale puisque "apprendre à se comporter à deux sous un même toit et constituer un espace commun, c'est construire l'espace de la relation en même temps que la relation. C'est construire un "nous" » (Eleb, 2004:313). L'espace habité renseigne ainsi sur le couple et dépeint, par l'entremise de nombreux indicateurs, le style conjugal de l'union qu'il abrite. Au cœur de ses agencements, de ses différents modes d'appropriation et des sociabilités qui se déroulent en son sein, se cristallisent en effet les négociations inhérentes à la relation du couple. L'habitat se présente ainsi comme un espace d'observation privilégié pour saisir et comprendre les modalités qui façonnent le style de la relation.

Cet article est issu d'une thèse de sociologie en cours, «Les parcours homosexuels et les styles de conjugalité chez les lesbiennes » (titre provisoire), sous la direction de Jean-Hugues Déchaux, université Lumière Lyon 2 - Centre Max Weber.

(1) Par le biais d'une étude de l'homoconjugalité féminine, la recherche doctorale en cours vise à saisir les processus par le biais desquels se construisent les couples lesbiens. En outre, par une comparaison des logiques conjugales observées chez les couples lesbiens et chez les couples hétérosexuels, cette recherche tente plus globalement d'étayer l'analyse de la diversité contemporaine des styles conjugaux. 
Les couples présentent, aujourd'hui, des modes de fonctionnement disparates. On observe, en effet, des unions "sur mesure " qui se caractérisent par la diversité des styles d'interactions qu'elles mettent en scène et par le rôle d'acteur que tient désormais l'individu dans le choix et la construction de sa relation conjugale (Kellerhals et al., 2004). La diversité des styles conjugaux contemporains " engage des philosophies conjugales distinctes, c'est-à-dire des manières de penser ce qu'est et doit être le couple» (Déchaux, 2007:34). Classifiés en cinq catégories, les styles conjugaux présentent des modes de cohésion et de régulation diversifiés. La cohésion renvoie au degré de fusion ou d'autonomie, au degré d'ouverture du couple à I'environnement et aux attentes des partenaires à l'égard de leur union. On observe, aux extrêmes, des configurations conjugales tantôt régies par la prépondérance des individualités, tantôt gouvernées par l'hégémonie du "nous conjugal ». La mesure de ces deux caractéristiques renseigne sur le degré de fusion ou d'autonomie qui orchestre la relation. "À cet égard, les philosophies conjugales sont diverses : certains pensent qu'un bon couple doit tout partager, et qu'il faut être d'accord sur tout ; d'autres, au contraire, veulent fixer au "nous couple" des limites précises et rester maîtres de ce qu'ils lui donneront ou non » (Kellerhals et al., 2004:57). La régulation se décompose en trois dimensions : la répartition ou la spécialisation des rôles dans le couple, la hiérarchie entre les genres et les générations et les règles instituant la vie du ménage.

Par l'étude des négociations conjugales qui se cristallisent dans les différents modes d'appropriation spatiale dans I'habitat, sont exposés dans cet article la spécialisation des rôles et le privilège accordé à l'autonomie ou à la fusion chez les couples lesbiens. À cet effet, I'analyse porte, en premier lieu, sur l'organisation qui sous-tend I'activité de décoration et le choix des ornements dans l'espace habité par le couple; elle est complétée par l'étude de la place accordée aux activités entreprises seules ou conjointement et par celle de l'agencement ou non d'espaces personnels dans I'habitat. Par le biais de l'ensemble de ces analyses, on s'intéresse au poids du "nous » couple et des « je » personnels dans l'organisation conjugale. Dans cette même dynamique, l'étude des sociabilités qui se déroulent au sein de cet espace permet de saisir les rapports plus ou moins ténus que ces couples entretiennent avec l'extérieur. L'observation s'attarde à cet effet, sur les modalités qui orchestrent l'ouverture ou la fermeture des couples à l'égard des cercles familiaux, amicaux ou de connaissances. L'analyse croisée de la configuration des réseaux de sociabilité et des modes d'ouverture de l'espace habité aux différents visiteurs montre la plus ou moins grande propension des couples à s'ouvrir sur l'extérieur. Enfin, l'association de ces différentes perspectives permet de dégager les différents styles de conjugalité qui s'expriment en tendance dans l'échantillon.

\section{Différents styles conjugaux}

Dans leur ouvrage Mesure et démesure du couple. Cohésion, crises et résilience dans la vie des couples $\left({ }^{*}\right)$ paru en 2004, Jean Kellerhals, Éric Widmer et René Levy déterminent cinq styles de conjugalité. La diversité des manières de faire couple n'apparaît pas, en effet, comme un ensemble anarchique mais se structure plutôt en quelques grandes tendances. Elle fait écho aux nouvelles contraintes contemporaines exigeant des individus d'établir leurs relations conjugales selon des modèles qui leur sont propres. Les cinq styles conjugaux présentent des organisations spécifiques au cœur desquelles prédominent des rôles, des valeurs, des priorités accordant un privilège plus ou moins prononcé à la routine, à la spécialisation, aux échanges avec l'environnement.

Le style «bastion » se caractérise "par son unité et son consensus plutôt que par la singularité ou l'autonomie de ses membres. L'organisation conjugale est agencée autour de différences de genre et de routines quotidiennes assez rigidement définies. Plutôt jaloux de son intimité, le groupe maintient cependant, par l'un de ses membres, un certain contact avec l'environnement. Les valeurs de solidarité, de stabilité et de sécurité dominent ». Le style " cocon" "s'organise autour des valeurs de confort. (...). Le groupe est clos sur lui-même. A I'intérieur du couple, les repères sont précis : les espaces sont dotés de fonctions précises et l'ordonnance des rythmes accentue le sentiment de sécurité. On n'est pas pourtant dans la tradition : le manque d'intérêt pour les fonctions externes de I'union ainsi que la faible différenciation des rôles fonctionnels et l'égalitarisme. (...) nous en séparent ». Le style " association » se caractérise par une "normativité assez faible. Les valeurs dominantes sont la négociation et la communication, moyens de concilier l'autonomie et l'authenticité reconnues à chacun avec les impératifs ou contraintes de la vie commune. (...). Le couple est très ouvert sur l'extérieur. II répugne aux routines et rejette une organisation du travail fondée sur la différence des genres". Le style " compagnonnage " met en scène des "individus qui s'estompent un peu derrière le souci du bien commun. L'envie de ne faire qu'un, mais aussi d'aller ensemble vers une communauté (...) à laquelle on souhaite rendre certains comptes, apparait comme le projet central. La souplesse en matière d'organisation conjugale, la volonté affirmée d'égalité et le refus de la routine s'orientent davantage vers l'idée de partage équitable que vers celle de négociation comptable ". Dans le style " parallèle ", "l'unité du groupe est bâtie sur la complémentarité des fonctions et la fermeture par rapport à l'environnement (...). La hiérarchie des genres est plus marquée qu'ailleurs et la routine est valorisée » (2004:74-82).

$\left.{ }^{*}\right)$ Kellerhals J., Widmer E. et Levy R., 2004, Mesure et démesure du couple. Cohésion, crises et résilience dans la vie des couples, Paris, Payot (réédité en 2008 dans la collection Petite bibliothèque Payot). 


\section{L'organisation conjugale dans l'appropriation spatiale}

Être chez soi, comme l'explique Yvonne Bernard, " C'est s'approprier un espace, le transformer en son bien » (Bernard, 1998:374). La notion d' « appropriation spatiale » est très importante. Roderick J. Lawrence la désigne comme "I'acte fondamental d'habiter» (Lawrence, 1982:71). Elle se décompose en de multiples dimensions. L'appropriation spatiale peut être d'ordre matériel et symbolique et se présenter à ce titre "comme la condition essentielle du chez-soi » (Mallon, 2004:371). Elle s'ancre également au cœur des enjeux identitaires et relationnels qui se jouent au sein des groupes familiaux. Certains auteurs ont travaillé "l'idée selon laquelle le logement est potentiellement un agent de régulation des relations familiales" (Bonvalet et Clément, 2005 ; Le Gall, 2005 ; Poittevin, 2005). Lieu de mise en scène de la vie quotidienne, I'habitat propose ainsi une lecture du jeu des interactions au sein du couple: "Pénétrer dans l'espace habité, c'est accéder à un lieu technique qui remplit des fonctions comme abriter, se nourrir, etc., mais aussi à un espace social et culturel chargé de signes distinctifs à découvrir et décrire » (Segalen et Le Wita, 1993:2). Tantôt collectives, tantôt individuelles, les appropriations spatiales qui se dessinent en son sein révèlent I'organisation conjugale, le dialogue entre le «je » de chaque partenaire et le «nous» conjugal.

Deux modes d'appropriation sont privilégiés dans I'analyse: l'activité de décoration et les activités conjointes et personnelles qui s'entreprennent au sein de l'habitat; sont ainsi analysés les négociations et les consensus conjugaux qui se matérialisent dans la décoration. Le couple trouve, en effet, à se définir et à se structurer dans ses meubles et ses objets (Eleb, 2004 ; Faure-Rouesnel, 2004 ; Kaufmann, 1991). Il s'agit d'explorer les pouvoirs décisionnels et d'influence qui participent de l'organisation conjugale et qui façonnent le décor des intérieurs des couples lesbiens. L'activité de décoration est, en outre, abordée sous l'angle de l'appropriation spatiale individuelle et collective qu'elle sous-tend. Le propos s'intéresse à cet effet aux expressions du «je » et à celles du «nous » qui s'inscrivent dans la décoration et souligne la prépondérance ou, au contraire, l'équilibre qui s'établit entre ces deux pôles. Enfin, I'analyse s'attarde sur l'alternance de la " vie seule » et de la "vie avec » qui se dégage des activités communes et personnelles entreprises dans l'espace habité, l'objectif étant de pointer le poids des individualités et de la conjugalité dans l'organisation conjugale. Dans cette lignée, est également entreprise une étude de l'agencement ou non d'espaces personnels (encadré, p. 68).

\section{Un profil conjugal dans la décoration}

L'étude de la décoration révèle des configurations disparates au sein des couples lesbiens enquêtés, accordant un privilège tantôt marqué tantôt ténu à l'inscription spatiale de la conjugalité. Dans la très grande majorité des couples, l'activité de décoration incombe à l'une des deux conjointes et n'apparaît que rarement partagée. Elle semble cependant toujours s'inscrire dans un mode spécifique de négociations conjugales. Quatre grandes tendances se profilent de ces négociations.

La première s'observe chez quatre couples; elle accorde la primauté à la conjugalité. Bien qu'entreprise par l'une des deux partenaires, la décoration reste l'affaire du couple. Le consensus et les concertations sont de mise, les pouvoirs décisionnels sont ainsi partagés. II revient à l'une des deux conjointes d'agencer ce qui a été préalablement discuté ensemble. Le choix des ornements et I'agencement du mobilier sont validés par les deux compagnes. La part des objets et des meubles acquis conjointement n'est pas négligeable et complète l'inventaire des effets appartenant initialement à chaque partenaire. Chez ces couples, la vie commune semble avoir favorisé la construction de goûts semblables facilitant aujourd'hui les consensus en terme de décoration. Chaque conjointe veille à ce que l'individualité de l'autre œuvre à l'expression du couple dans le décor de l'habitat. Des qualités respectives sont reconnues : à l'une le sens pratique et à l'autre le sens esthétique, à l'une les qualités de " designer » et à l'autre la minutie de la décoration. Pour autant, l'équilibre entre les individualités et la conjugalité bénéficie d'un certain intérêt. On agence, dans le décor, des objets personnels et d'autres acquis en couple. Le mélange des effets personnels est toutefois chargé d'une dimension conjugale.

- Clémence : C'est surtout Tatiana qui décide du choix des objets de déco. Moi, c'est vrai que j'interviens peu parce que j'ai peu d'idées ; je ne suis pas très bonne là-dedans. J'aime ce qui est beau, je donne mon avis sur ce que j'aime et ce que je n'aime pas mais c'est vrai que, les objets de décoration, c'est plutôt à l'initiative de Tatiana.

- Enquêtrice : Et ça ne te dérange pas?

- Clémence : Non parce que c'est un petit peu sa partie à elle, c'est là où elle se sent bien, c'est là où elle aime s'exprimer. Elle a de très bons goûts; généralement, ce qu'elle décide ça me plaît, donc je me fie à son choix parce que, si elle me mettait ça dans les mains, je ne saurais pas quoi en faire ; donc ça me va très bien comme ça. (...). Généralement je m'occupe du côté pratique-rangement, très basique, et Tatiana choisit et module les choix selon le côté esthétique parce qu'elle a plus de vision et d'imagination que moi pour imaginer ce qui va être bien et pas bien au niveau de la forme, etc. 


\section{Méthodologie}

\section{Les entretiens}

Cette enquête consiste en vingt-huit entretiens approfondis. Ces derniers ont été menés auprès de quatorze couples lesbiens et constituent une partie du matériau de la recherche doctorale en cours. Décomposés en deux parties - l'une consacrée au récit du parcours homosexuel et conjugal et l'autre à l'organisation de la vie quotidienne dans l'espace habité par le couple -, ils ont été entrepris auprès de chaque conjointe. Le contact avec les enquêtées s'est établi par l'intermédiaire de différents réseaux de connaissances.

Organisée autour d'une série de questions volontairement larges et inaugurales, la première partie d'interview emprunte à la méthode du récit de vie et ne fait pas I'objet d'investigations pour cet article. Les résultats rapportés se dégagent, en effet, de la seconde moitié qui, structurée autour d'un guide d'entretien plus directif visait à aborder les pratiques et les représentations participant de l'organisation conjugale dans l'habitat. L'étude s'est intéressée à ce propos à l'intégration du couple dans la famille et dans les sphères amicales et professionnelles de chaque conjointe, à l'organisation domestique, aux sociabilités dans I'habitat, aux ouvertures et aux fermetures du logement, à son organisation spatiale et aux appropriations conjointes et/ou personnelles dont il pouvait faire l'objet. Les entretiens individuels menés au domicile conjugal ont présenté plusieurs intérêts. Ils ont tout d'abord facilité la passation et la libre expression. Par ailleurs, ils ont favorisé les discussions et les échanges sur le décor et les ornements de décoration. Nombre des réponses fournies par les interviewées ont été illustrées par un recours à la matérialité dans l'habitat, par un objet pris en exemple et qui, $d^{\prime}$ apparence souvent anodine, se révélait être une précieuse source d'informations.

Ces entretiens individuels ont également permis la confrontation du discours des deux conjointes. En effet, au regard de la perception et de l'évaluation différenciées que chaque partenaire se fait des choix pourtant effectués en couple, "on s'aperçoit souvent qu'habitant ensemble, mentalement [les conjoints] ne vivent pourtant pas dans le même espace » (1). Aussi, bien que la perspective présente de nombreux intérêts sociologiques, il ne s'agissait pas, dans les démarches empiriques, de rechercher "l'interaction triangulaire, créée par l'entretien "conjoint" » et de plonger au cœur des mises en scène conjugales. Pour reprendre le propos d'Isabelle Clair : "Se montrer en couple à un tiers n'a pas la même signification que de parler de son couple individuellement : I'entretien "conjoint" est intimement lié à l'interaction conjugale qui se déroule en même temps (...). Certes, qu'un couple se donne à voir de la sorte est en soi une information sur son mode de fonctionnement, sert l'analyse. Mais c'est aussi un écran à la compréhension de sa réalité quotidienne puisque c'est celle-ci, plus encore que la réalité de l'entretien, qui nous occupe» (2). Afin de saisir I'organisation conjugale, il fallait donc se approcher au plus près du quotidien des interviewées, de la répartition des tâches et de l'ordonnancement des rôles. Dans cette perspective, la confrontation des réponses émises par les deux partenaires lors du questionnement détaillé de l'organisation domestique a été systématique. En pointant les écarts, il s'agissait de neutraliser les effets de mise en scène de soi et de son couple.

\section{La population enquêtée}

La sélection des couples s'est en partie effectuée par l'âge des partenaires. Face à la contrainte d'une éventuelle variation d'âge entre conjointes et afin de minimiser les effets de génération, un seul critère a été retenu.
Chaque conjointe devait être âgée de plus de 25 ans et de moins de 40 ans. La moyenne d'âge se situe autour de 32 ans. Au regard des travaux menés sur les parcours homosexuels et conjugaux, on a en effet estimé que, au-delà de 25 ans, les probabilités d'être investi dans une relation stable et durable seraient plus importantes. Outre le critère d'âge, chaque couple devait justifier de plus d'un an de vie commune dans un même logement et ne pas avoir d'enfant(s). L'habitat et la localisation résidentielle ont fait l'objet d'une attention particulière. Afin de faciliter la recherche d'enquêtées et de neutraliser l'effet des localités géographiques sur les modes de vie, il s'agissait de sélectionner, dans la mesure du possible, des couples habitant un même type de logement (de deux pièces ou de trois pièces) dans un espace urbain. Enfin, les appartenances sociales apparaissent diversifiées au sein de la population enquêtée. Alors que l'on observe, dans les recherches menées sur I'homoparentalité, une plus grande propension des femmes les plus diplômées à se raconter et à ouvrir leur sphère familiale (3), on relève dans l'échantillon une proportion non négligeable de femmes faiblement qualifiées. Ces femmes les plus en proie à la précarité et à la stigmatisation manifestaient une forte envie de se raconter et le besoin d'être reconnues dans leur couple et leur vie quotidienne. En outre, les critères d'âge retenus pour l'échantillon associés au choix d'enquêter des couples sans enfant semblent avoir encouragé et orienté les contacts avec ses femmes les moins qualifiées. À cet égard, la diversité des réseaux et des espaces géographiques au sein desquels s'est entreprise la recherche d'enquêtées annule la probabilité "d'un effet de réseau ». Au regard de la configuration de l'échantillon, est ainsi formulée l'hypothèse que le passage du couple à la famille pourrait être conditionné par certaines ressources sociales, ressources dont seraient dépourvues les femmes les plus exposées à la précarité. Cette hypothèse pourrait, en partie, expliquer la plus grande proportion d'enquêtées appartenant aux catégories favorisées dans les recherches traitant de l'homoparentalité. Parmi les couples lesbiens rencontrés, l'hétérogamie non négligeable (puisqu'elle concerne neuf couples sur quatorze) est venue contrarier toute tentative d'homogénéisation des appartenances sociales. II n'existe aucune statistique traitant des caractéristiques sociales et de la structuration du marché matrimonial au sein des populations lesbiennes. L'écart de niveau d'instruction au sein de ces couples apparaît lui aussi signifiant puisqu'il se retrouve chez sept couples. Toutefois, une certaine homogamie sociale aux extrêmes de la composition de la population est observée. Cette homogamie regroupe, à un pôle, des femmes occupant un emploi hautement qualifié et, à l'autre pôle, des femmes peu voire non diplômées, justifiant d'un emploi faiblement qualifié. L'homogamie semblerait ainsi s'observer au sein des catégories sociales supérieures et au sein des classes populaires sous-qualifiées. Entre ces deux pôles, le niveau de qualification et les professions et catégories socioprofessionnelles apparaissent disparates au sein des couples (4).

(1) Eleb M., 2004, Le roman du couple et sa scène spatiale, in Espaces domestiques (sous la dir. de Collignon B. et Staszak J.-F., Paris, Bréal:311.

(2) Clair I., 2007, Dire à deux le ménage, in L'injustice ménagère [sous la dir. de Singly F. (de)], Paris, Armand Colin:209.

(3) Descoutures V., 2010, Les mères lesbiennes, Paris, PUF.

(4) Voir le tableau page 77 qui propose une lecture rapide des résultats d'analyse présentés dans I'article et des principales caractéristiques sociales de la population enquêtée. 
- Enquêtrice : Et dans la manière dont c'est agencé, vous vous êtes concertées ou c'est Tatiana qui a plutôt mis en place les choses?

- Clémence : Non, là, on s'est concertées; on a décidé ensemble de ce que l'on mettrait où et comment. Mais toujours dans la même logique, c'est-à-dire qu'elle, elle voyait ce qui était esthétique, et moi je voyais le côté pratique.

- Tatiana : Les objets de déco c'est plus moi, c'est moi qui ai peut-être plus le feeling et plus le... Clémence est beaucoup plus pratique et moi je suis beaucoup plus dans l'esthétique. (...) Je pense qu'elle a tendance à me faire confiance. Elle me laisse faire un peu. Par contre, au niveau des finances c'est clair que c'est elle qui gère, c'est elle qui fait les budgets (...).

- Enquêtrice: Comment a été agencé le salon? Comment vous vous êtes organisées?

- Tatiana: On a tout négocié, enfin c'était tout dans la discussion, on a toujours discuté de tout en fait, "qu'est-ce que tu en penses?", "Oui mais, attends, là c'est peut-être mieux", "attends on va essayer". Oui ça a toujours été fait comme ça, dans la concertation.

La deuxième tendance s'exprime chez cinq couples. La décoration incombe à l'une des deux conjointes mais ne fait pas l'objet de consensus. L'exclusivité du pouvoir décisionnel dans le domaine apparaît tantôt imposée tantôt négociée dans le couple. Lorsque l'une des deux conjointes cède l'activité de décoration par conciliation, un accord s'établit autour du partage des tâches. La manière d'agencer et de décorer ne fait pas I'unanimité mais on évite les discordes par une répartition des tâches qui fait consensus. Les conjointes ne participant pas à la décoration arguent d'un moindre intérêt pour l'activité et expliquent la concéder pour satisfaire les attentes de l'autre. Des désaccords s'expriment lorsque ni la décoration ni la répartition des tâches n'a été approuvée par chaque conjointe. Dans tous les cas, les goûts en matière de décoration n'apparaissent que faiblement partagés et le choix des objets exposés dans l'habitat ne revient qu'à I'une des deux partenaires. On relève alors dans le décor I'expression d'une individualité prépondérante. La sélection des ornements n'est pas discutée et la décoration se compose, pour beaucoup, des effets personnels de la conjointe qui décore. La conjugalité n'est ainsi que faiblement matérialisée.

- Enquêtrice: D'une manière générale, c'est quand même toi qui prends plus en charge la déco?

- Carine : Oui.

- Enquêtrice : parce que Julie ne s'y intéresse pas...? - Carine : On va dire qu'elle m'a cédé la déco et, par contre, pour d'autres choses, c'est elle qui prend en charge.
- Enquêtrice : C'est parce que la déco ne l'intéresse pas forcément?

- Carine : Si, pourtant, le pire c'est que c'est un petit peu proche de son métier. Elle travaille dans les rideaux, tout ça, dans la déco, mais c'est quelque chose qui me tient à cœur et elle le sait, donc elle a passé la main dessus (...). C'est vrai que là-dessus quand même elle me laisse faire. Les statues, pratiquement tous les objets de déco ici, elle ne les aime pas. Mais elle comprend que je les garde parce que, moi, je trouve que ça crée une ambiance, ça crée une atmosphère et, puis, j'ai besoin de tout ça, de ces trucs zen; et elle a accepté mais je sais qu'elle déteste, ça je sais qu'elle n'aime pas.

- Enquêtrice : Et, à l'inverse, est-ce qu'il y a des objets à elle que tu n'aimes pas du tout?

- Carine : Oui, son cadre là-haut, je n'aime pas du tout, surtout que je trouve qu'il n'est pas du tout en accord avec ma déco [rires] mais, bon, comme c'est un cadeau de son papa, c'est quelque chose qui lui tient très à cœur; on l'a mis au centre de la salle à manger.

- Julie : La déco c'est plus elle, je dirais que c'est plus son côté à elle, si elle veut tout changer tant mieux [rires], si elle veut laisser tant pis.

La troisième tendance se distingue chez trois couples. Elle se caractérise par l'intervention des deux partenaires dans le travail de décoration. Dans un premier cas, I'activité de décoration et les goûts en matière d'ornements reviennent à l'une mais restent soumis aux exigences de l'autre. Ces exigences peuvent être d'ordre pécuniaire. L'apport financier de celle qui dispose du plus gros revenu confère alors un certain droit de regard sur l'achat des éléments de décoration et sur le budget qui lui est imparti. Ce droit de regard s'exprime d'autant plus fortement que les frais de décoration incombent à la conjointe qui ne décore pas. Autre situation possible, la décoration peut être entreprise par les deux conjointes de manière séparée. Chaque partenaire contribue alors à édifier le décor intérieur sans concertation préalable. On relève toutefois un consensus autour des goûts partagés. Enfin, on note l'absence de consensus et de goûts communs dans le choix des ornements et l'envie chez chaque conjointe de participer à la décoration. Le décor intérieur se présente alors comme un véritable champ de négociations. On opte pour le « donnant-donnant » et pour le partage du territoire. Les négociations n'empêchent pas que I'une des partenaires bénéficie dans le domaine d'un pouvoir décisionnel plus marqué. Dans l'ensemble de ces cas de figure, s'exprime la cohabitation de deux individualités dans le décor de l'habitat. La part des objets acquis conjointement reste faible et laisse place aux effets personnels des deux conjointes dont le mélange n'exprime pas la conjugalité. Le couple 
ne trouve ainsi à s'inscrire que faiblement dans la décoration.

- Delphine : Les objets de déco qui, pour l'instant, sont entreposés ici sont essentiellement à moi. La déco, c'est moi qui m'en occupe et ça se négocie parce que j'ai des goûts de déco qui sont fondamentalement différents de ceux de Raphaëlle (...). Raphaëlle, elle va plus me freiner par rapport à mes envies de bougeoirs que je mettrais partout. Après, je reste une nana qui est persuasive et quand je veux vraiment quelque chose je sais l'obtenir. Et puis je pense que, quand elle a vraiment envie de quelque chose, elle sait y faire et elle l'obtient aussi. Je crois que ça fait partie du jeu.

- Enquêtrice : Ça t'embête de devoir négocier?

- Delphine: Non, pas plus que ça.

- Raphaëlle : Elle a plein de machins où elle met ses bougies, je trouve ça vraiment laid mais, après, c'est affaire de goûts. Moi je ne suis pas bibelots, pas du tout ; j'aime la technologie (...). C'est pareil, les miroirs ce n'est pas son truc. Pour moi, les miroirs ça agrandit la pièce, ça fait plein de choses, ça fait entrer de la lumière, enfin voilà (...). Il y a déjà des négociations, il y a déjà du travail au corps. J'aurai droit à plusieurs choses pour qu'elle, elle ait son truc.

La dernière tendance s'observe chez deux couples ; elle se caractérise par un faible investissement dans la décoration et par une mise en avant de la fonctionnalité de I'agencement spatial. On distingue toutefois quelques éléments de décoration appartenant à l'une ou à l'autre des conjointes et une expression parcimonieuse et cloisonnée de chaque individualité dans les lieux. L'absence d'intérêt pour la décoration et pour l'acquisition d'objets communs souligne la moindre importance accordée à l'inscription du couple dans le lieu de vie. Pour l'un des deux couples, I'importance accordée au caractère fonctionnel de l'agencement dissimule des désaccords quant aux choix des objets de décoration et des goûts disparates entre les conjointes. La fonctionnalité des lieux et l'absence de décoration permettent alors de préserver la neutralité des espaces et d'échapper aux discordes. La décoration ne porte ni l'empreinte du couple ni l'empreinte de l'individualité de chaque conjointe.

- Claudine : On n'a pas énormément de déco, on n'a pas eu le temps, en fait, de s'en occuper. C'est plus de l'utile qui a été installé au fur et à mesure, sinon le peu de déco qu'on a... au salon, on a que quelques tableaux mais...c'est vrai qu'on n'a pas encore trop engagé ce genre de...parce que, là, du coup, il faut réussir à trouver un compromis et ce n'est pas évident.

- Enquêtrice : Ah oui, pourquoi?

- Claudine : Parce qu'on n'a pas les mêmes goûts, c'est évident, donc il faut trouver des tableaux ou des... il faut mettre sa marque sans trop la mettre parce qu'on n'est pas... parce qu'on a envie que ce soit un endroit commun. C'est peut-être la différence qu'il y a d'ailleurs entre un couple lesbien et un couple hétéro finalement. Je pense que les femmes hétéro se permettent plus de créer la déco dans la maison du couple alors que, nous, pas tant que ça parce que, pour le respect de l'autre, c'est vrai qu'on donne peut-être plus la place au compromis finalement (...).

\section{La conjugalité entre "vie seule » et "vie avec "}

L'étude des investissements conjoints et personnels des différents espaces de l'habitat permet d'étayer I'analyse des organisations conjugales. Elle vise à saisir I'alternance entre "la vie personnelle et la vie commune» qui se profile dans l'usage des espaces habités; "la vie privée nous apprend que, le bien vivre ensemble est tout autant défini par les phases où chaque membre du groupe vit dans son monde personnel que par les moments de partage commun " [Singly (de), 2008:22]. Au regard de la diversité des styles de conjugalité contemporains, les modalités qui régulent la «vie seule» et la " vie avec » sembleraient relever d'une organisation conjugale plus ou moins fusionnelle. L'analyse de la répartition des temps de vie commune et séparée dans I'habitat apparaît, en effet, heuristique pour appréhender la prééminence du couple ou de I'autonomie individuelle dans la vie quotidienne. Ainsi, l'exposé s'attarde sur le privilège accordé aux activités conjointes et personnelles lorsque le couple est réuni dans l'habitat. L'observation s'attarde sur les besoins et les modes d'isolement des partenaires ainsi qu'à la répartition homogène ou non de l'investissement des partenaires dans les différentes pièces de l'habitat. Elle se focalise, enfin, sur l'agencement d'espaces personnels et sur la nécessité qu'expriment les conjointes à cet égard. Les modalités qui pondèrent la «vie seule » et la «vie avec » montrent des configurations hétérogènes chez les couples lesbiens enquêtés. Là encore, plusieurs tendances peuvent être rapportées.

La première, qui s'observe chez six couples, se caractérise par une forte valorisation des activités et des moments partagés. Le privilège n'est pas accordé aux activités personnelles qui, lorsqu'elles sont effectives, semblent être entreprises aux côtés de l'autre conjointe. La vie quotidienne est d'abord définie par "la vie avec ». Chez ces six couples enquêtés, la retraite temporaire et le besoin d'isolement sont faibles. Le retrait dans une pièce à part lors d'une conversation téléphonique n'est pas de mise et reste déprécié au sein du couple. La vie quotidienne s'associe ainsi fortement au caractère conjoint des activités partagées dans l'espace habité. 
L'hégémonie du " nous » conjugal se retrouve dans le décor de trois des six couples ici concernés.

- Enquêtrice : Est-ce qu'il y a des pièces où il t'arrive d'être seule quand Laura est là?

- Juliette : Oui, un peu la salle de bains, un peu la cuisine si je me mets à cuisiner et qu'elle veut rester tranquille mais, en principe, on se suit pas mal nous en fait. Donc si je me mets à cuisiner et que c'est le soir et qu'on ne s'est pas vues de la journée, elle va s'installer avec moi dans la cuisine. Je dirais que c'est plus la salle de bains et, encore, on la partage assez souvent (...). Le moment où je vais chercher à m'isoler c'est plutôt un temps où, au départ, je suis seule. Je cherche rarement à m'isoler quand on est ensemble. J'ai besoin d'être avec l'autre même si je ne partage pas à ce moment-là.

- Enquêtrice: Est-ce qu'il t'arrive de t'isoler lors d'une conversation téléphonique?

- Juliette : Non.

- Enquêtrice : Est-ce qu'il t'arrive parfois de t'isoler au sein de l'appartement?

- Laura : Rarement.

- Enquêtrice: Est-ce qu'il t'arrive de t'isoler lors d'une conversation téléphonique?

- Laura: Rarement, c'est plus dans l'idée de ne pas déranger quand Juliette regarde la télé, bouquine, ou soit parce que je sais que ça va durer longtemps.

Le deuxième ensemble regroupe deux couples. II se définit par la primauté des activités personnelles entreprises dans une pièce inoccupée. Les conjointes rapportent ainsi l'importance des activités auxquelles elles s'adonnent seules dans une pièce qui n'est pas investie par l'autre partenaire. Les investissements spatiaux semblent fortement individualisés. Le salon est convoité par l'une et le bureau par l'autre. Le besoin de s'isoler et la nécessité de disposer de temps et d'un espace à soi font consensus au sein du couple. On remarque l'agencement d'un espace personnel situé dans une pièce réservée à cet effet pour au moins une des deux conjointes. La propriétaire des lieux y trouve un espace pour s'adonner à ses travaux professionnels ou artistiques. Elle y assouvit également son besoin d'isolement. Chez ces deux couples, la décoration de l'habitat ne porte l'empreinte ni de la conjugalité ni des individualités.

- Enquêtrice: Quelle est la pièce où tu passes le plus de temps?

- Cécile : Là [dans le salon].

- Enquêtrice: Tu y fais quoi?

- Cécile : Je suis devant la télé ou sur l'ordinateur (...). Amandine est souvent dans le bureau à travailler sa musique. Je vais dans la chambre aussi parce que j'y dors souvent les après-midi ; donc, quand je fais la sieste ça dure bien trois ou quatre heures. Mais je pense que c'est cette pièce [le salon].

- Enquêtrice: Est-ce qu'il t'arrive de t'isoler lors d'une conversation téléphonique?

- Cécile : Ça m'arrive oui parce que, par exemple, Amandine amène son portable ici et bosse là, et moi je suis assise à mon ordinateur ; je parle et elle a tendance à me dire que je parle trop fort, donc, du coup, je vais me mettre dans le bureau. - Enquêtrice : Est-ce qu'il y a une pièce dans laquelle tu passes plus de temps que Cécile?

- Amandine : Oui, le bureau.

- Enquêtrice : C'est un espace à toi le bureau?

- Amandine : C'est le lieu où je peux m'isoler pour travailler, donc je me suis un petit peu accaparé le lieu; mais elle y va aussi parce qu'il y a des trucs. C'est une chambre à la base, normalement on y fait dormir les amis. C'était une chambre et j'en ai fait un bureau.

- Enquêtrice : C'est important d'avoir cet espace-là pour toi?

- Amandine : Oui, parce que parfois j'ai besoin de me retrouver avec moi, comme Cécile a besoin aussi de se retrouver de temps en temps. Cécile, ça sera plutôt le salon, elle y a son ordinateur, et la télé. Et moi ça sera plutôt le bureau parce que j'ai mon instrument de musique et que c'est là aussi que j'ai mon bureau et que j'ai mes feuilles pour écrire. Je suis en tête à tête avec moi; d'ailleurs quand je suis dans le bureau je ferme la porte. C'est rare que je laisse ouvert; si je laisse ouvert c'est qu'elle peut venir. Si je ferme, c'est que je vais être occupée, affairée et que j'ai besoin de mon petit espace.

La troisième tendance s'observe chez six couples et se caractérise par une valorisation des activités entreprises seule et en binôme. L'importance accordée aux activités conjointes ne minimise pas celle des activités entreprises seule. L'alternance des temps de vie commune et séparée contribue, dans les discours, à l'équilibre du couple et au respect des individualités. Chaque conjointe semble faire usage de I'habitat dans son ensemble. L'absence d'espace personnel est éloquente. Le besoin d'isolement, exprimé par toutes les enquêtées, ne conduit pas à l'agencement d'un espace réservé à cet effet. On se retire dans une pièce inoccupée ou on trouve à s'isoler par le biais d'une activité entreprise en la présence de l'autre. Le caractère privé des conversations téléphoniques fait consensus dans le couple et justifie le retrait dans une pièce à l'écart. On veille à respecter l'intimité personnelle. Le décor intérieur des six couples concernés ne réifie que faiblement la conjugalité. II porte essentiellement la marque d'une ou de deux individualités. 
- Enquêtrice : Est-ce qu'il t'arrive de t'isoler dans I'appartement?

- Jeanne : Ah oui, oui ça nous arrive souvent. Ça arrive même des jours où on ne se voit que pendant les repas et le reste de la journée on ne s'est pas vues parce que Justine avait envie de lire et moi j'avais envie de regarder un film.

- Enquêtrice: C'est des moments... C'est important pour vous de pouvoir...?

- Jeanne : Ah oui, c'est nécessaire, je crois que ça fait partie de la condition essentielle à l'équilibre d'un couple. Je crois que c'est essentiel, on ne peut pas être toujours... et puis on n'a pas les mêmes envies tout le temps, je crois que c'est indispensable (...).

- Enquêtrice : Est-ce qu'il t'arrive de t'isoler lors d'une conversation téléphonique?

- Jeanne: Oui ça peut arriver, ou alors j'appelle quand elle n'est pas là.

- Enquêtrice : Ça rejoint l'idée de pouvoir faire des activités dans des pièces séparées?

- Jeanne : Oui ou c'est des gens que je n'ai pas vus depuis longtemps qui vont me demander des trucs sur notre vie et que, des fois, je préfère... Je n'ai pas de trucs à lui cacher mais des fois je me sens plus à l'aise si elle n'est pas forcément à côté de moi. Comme elle, par exemple, quand elle appelle son meilleur ami, elle vient sur le canapé quand je suis dans le bureau. Ça ne me pose aucun problème, je crois qu'il y a des moments où elle a besoin de se sentir toute seule avec la personne avec qui elle est au bout du fil.

- Justine : Oui ça nous arrive d'être l'une dans le bureau et l'autre dans la chambre parce qu'on ne va pas faire la même chose en même temps ou qu'on a besoin d'être tranquille, qu'on n'a pas spécialement envie de parler.

- Enquêtrice : Est-ce que, toi, tu disposes d'un coin à toi ou d'un endroit à toi ?

- Justine : Pas spécialement, non.

- Enquêtrice : Tu aimerais en avoir un?

- Justine : Non parce qu'on arrive à justement se laisser du temps et à accepter que l'autre se mette dans une pièce parce qu'elle a envie d'y être tranquille.

À la croisée des investissements matériels et des activités qui s'entreprennent au sein de l'habitat, se profilent ainsi des styles de conjugalité différenciés. En effet, s'expriment en filigrane différentes manières de faire couple, avec un primat plus ou moins prononcé au «nous » conjugal. On observe, à un pôle, une cohésion centrée sur le couple. Les individualités s'estompent dans un décor intérieur qui affiche la mise en scène conjugale. Les expressions individuelles ne sont que faiblement valorisées et sont mises à profit du couple lorsqu'elles sont effectives. Les négociations conjugales bénéficient $d^{\prime}$ 'un intérêt premier et semblent orchestrer la répartition des pouvoirs décisionnels dans le couple. On relève, à l'opposé, une cohésion régie par les deux individualités qui forment alors un binôme conjugal. Cette configuration reste largement minoritaire; elle ne concerne que deux couples sur quatorze. La primauté semblerait être accordée au « je » et le couple resterait au service de I'individu. L'organisation conjugale rappelle alors la "quasi-conjugalité » que Jean-Claude Kaufmann relève chez les jeunes couples parvenant à "rester en situation de cohabitants sans intégration, l'organisation légère et séparée des tâches ménagères permettant de ne pas engager l'avenir, l'inexistence de trop lourdes habitudes collectives laissant le choix du conjoint à l'état nu, fondé sur le seul sentiment" (Kaufmann, 1992:73). Entre les deux extrêmes, les organisations conjugales affichent un certain équilibre entre le «je » et le "nous ». Toutefois, cet équilibre n'aboutit pas à des configurations parfaitement homogènes. II s'exprime en tendance et sous-tend parfois une inclination légèrement plus prononcée pour le couple ou pour les individualités. La décoration et le pouvoir décisionnel dans le domaine ne font pas toujours consensus même si les tentatives de négociation sont effectives. Les activités affichent un caractère tantôt personnel, tantôt collectif. La fusion, prédominante dans la première configuration conjugale, n'est pas de rigueur dans celle-ci.

La pluralité des organisations conjugales qui s'exprime dans la gestion de la décoration ne démontre ni une plus grande propension à la négociation ni un égal rapport à la décoration chez les couples lesbiens. Cette pluralité rappelle la diversité des styles conjugaux chez les couples hétérosexuels. La répartition selon le genre des tâches qui contribue à caractériser les styles hétéroconjugaux les plus traditionnels semble ne pas laisser place à davantage de partage au sein des unions féminines. L'activité de décoration revient, par exemple, chez plusieurs couples lesbiens, à celle qui exprime un plus grand intérêt en la matière ou encore à celle à qui l'on reconnaît les compétences requises.

\section{L'ouverture et la clôture du couple et de son habitat}

L'habitat contient l'intimité conjugale et le caractère à la fois privé et public de l'union qu'il abrite. Il s'inscrit au carrefour d'une articulation de la vie privée et de la vie publique (Prost, 1999); "Le logement est le lieu par excellence de l'intimité domestique. Mais il constitue également un pôle important de la vie relationnelle des individus » (Authier, 2001:53). Il y a des espaces semipublics, dans lesquels se nouent et s'entretiennent les liens avec l'extérieur, et des espaces privés empreints de l'intimité des occupants et tenus, à ce titre, fermés aux visiteurs. Comme le rappelle 
Laurence Faure-Rouesnel, " outre cette dimension privative qui est consubstantielle au logement, l'espace domestique ne se résume pas à un espace privé. Bien que vécu au quotidien par la famille, le logement est, en effet, sans cesse pénétré et traversé de la vie du dehors, de l'espace public et de la société dans son ensemble » (Faure-Rouesnel, 2006:207). Par les sociabilités qui se déroulent en son sein, par ses modes d'ouverture et de fermeture à autrui, le logement renseigne sur la privatisation et la publicisation de la relation conjugale. En effet, "s'intéresser aux degrés d'ouverture de la maison est aussi un moyen d'appréhender (...) la cohésion et l'identité du ou des groupe(e)s constitué(s) dans la maison»(Gherchanoc, 2006:26). Aussi, afin d'étayer l'étude du caractère plus ou moins fusionnel des couples lesbiens enquêtés, dans la suite de l'article sont analysés la fréquence et les différents types de sociabilités privilégiés dans les lieux.

\section{Des réseaux de sociabilité hétérogènes}

Les échanges interpersonnels privilégiés dans I'habitat sont mobilisés comme autant d'indicateurs d'ouverture du couple sur l'extérieur. Quelle est la propension des couples à ouvrir ou, au contraire, à fermer l'espace qu'ils investissent de leur relation? Cette propension est étudiée au regard du degré de proximité qu'ils entretiennent avec les personnes conviées dans l'habitat. L'étude de l'ouverture du couple ne saurait s'établir sans une analyse préalable des rapports entretenus avec les environnements. Aussi, afin d'apprécier l'inclination plus ou moins forte au repli domestique, l'observation s'attarde sur les contacts familiaux et sur la configuration des réseaux amicaux et de connaissances. Par le biais du degré de proximité que les couples partagent avec leurs visiteurs, il s'agit d'exposer l'aptitude plus ou moins grande à ouvrir son intimité conjugale. Quant à l'analyse de la composition des cercles amicaux et de connaissances, elle permet de saisir la répartition du rôle de pourvoyeur de relations dans l'organisation conjugale.

À l'instar de la définition proposée par Yves Grafmeyer, les sociabilités sont entendues ici comme des rapports interpersonnels, hérités ou construits, qui se caractérisent par un minimum d'intensité, de régularité et de stabilité au cours du temps. Toutefois, traiter de la sociabilité amène également à s'intéresser aux interactions plus éphémères et plus fragiles qui peuvent s'instaurer en diverses circonstances de la vie quotidienne (Grafmeyer, 1995). Les analyses se sont ainsi intéressées aux sociabilités amicales et familiales impliquant des liens forts, hérités ou construits et aux sociabilités relevant de "simples » connaissances mobilisant des liens faibles et plus fugaces.

Les réseaux de sociabilité montrent de fortes disparités chez les couples lesbiens enquêtés. Tout $d^{\prime}$ abord, les contacts que les couples entretiennent avec leurs cercles familiaux apparaissent hétérogènes. Ils se diversifient tant par leur fréquence que par le caractère des relations qui les motivent. Ils ne se présentent toutefois que faiblement heuristiques pour l'analyse de l'ouverture des couples sur l'extérieur. Les relations à la famille demeurent, en effet, fortement soumises à la tolérance exprimée par les proches à l'égard de I'homosexualité. Alors que cette dernière n'est tue chez aucune des lesbiennes interviewées, son acceptation ou non par la famille s'accompagne de situations plus ou moins conflictuelles. Les cas de rupture familiale sont minoritaires; ils s'observent chez deux enquêtées. En outre, bien que toujours effectifs, en réponse à l'hostilité parentale, les liens peuvent être distendus et les sociabilités familiales entretenues hors du domicile conjugal. Ce cas de figure se rencontre chez cinq interviewées. Enfin, dans la très grande majorité des situations, l'habitat se définit avant tout comme l'espace des sociabilités amicales et ne se trouve mobilisé pour les échanges familiaux que dans une moindre mesure. Alors qu'après "vingt-neuf ans, à la formation du couple [hétérosexuel], la part de la sociabilité amicale décline très fortement " et que, "dans un réseau globalement plus restreint, les relations familiales plus hétérogènes en âge prennent alors un poids plus important ", les relations amicales prédominent parmi les sociabilités des couples lesbiens (Rissoan, 2004:149). Les contacts familiaux, moins fréquents, s'entretiennent principalement au domicile parental. Parmi les couples, les réseaux amicaux et de connaissances tendent à se différencier par leur taille et leur configuration. Leur amplitude varie selon que les contacts avec l'extérieur sont entretenus par l'une des conjointes ou les deux. On relève dans leur composition le privilège plus ou moins grand accordé à I'homosociabilité (2).

\section{Des types de sociabilité pour des styles de conjugalité}

$\mathrm{Au}$ regard de l'étendue, de la composition des réseaux, de la fréquence et des types de sociabilité qui s'entretiennent dans l'habitat, on observe une classification de l'ouverture ou de la clôture des couples en quatre tendances.

La première caractérise des couples très ouverts sur l'extérieur. Ces derniers nourrissent des échanges au sein du cercle d'amis et de connaissances qu'entretient chaque conjointe. La taille et l'importance

(2) L'homosociabilité renvoie ici aux manières d'être et d'agir, aux goûts et aux intérêts qui guident les échanges et les pratiques au sein d'un groupe amical composé exclusivement d'individus homosexuels. 
de ces réseaux répondent aux activités professionnelles et aux investissements associatifs de chacune. Les rôles de pourvoyeur de relations et les contacts avec l'extérieur sont ainsi partagés. Les réseaux se composent d'amis de l'une et de l'autre mais comprennent aussi pour partie des amis et des connaissances rencontrés conjointement lors de sorties ou d'activités entreprises en couple. L'habitat contribue fortement au maintien de ces relations sociales. Il est ouvert aux amis et aux personnes avec lesquelles n'est entretenu qu'un faible degré de proximité. Les réceptions sont fréquentes et font l'objet d'un intérêt particulier. On valorise les échanges et la diversité des contacts au domicile. Les visites peuvent être programmées mais aussi spontanées. Leur promptitude et la sélection plutôt lâche qui s'opère à l'entrée de l'habitat soulignent la propension à I'ouverture. Les cercles d'amis et de connaissances affichent une mixité homosexuels-hétérosexuels. La proportion d'homosexuels apparaît toutefois plus importante au sein des réseaux de connaissances, en raison, pour l'essentiel, de la fréquentation des bars, des discothèques et des associations gays et lesbiennes. Pour autant, le mélange d'homosexuels et $d^{\prime}$ 'hétérosexuels au domicile et dans les sociabilités fait l'objet d'une valorisation. Au regard des analyses précédentes, on remarque que I'organisation de ces trois couples se caractérise par une place non négligeable accordée aux individualités. Au profit du couple, les réseaux relationnels distincts de chaque conjointe portent la marque des individualités.

- Enquêtrice: Est-ce que vous avez le sentiment d'avoir un réseau de connaissances important?

- Claire : Oui quand même.

- Enquêtrice : Et parmi ces connaissances, certains ou certaines sont homosexuel-le-s?

- Claire : Oui.

- Enquêtrice : Plus que parmi les amis?

- Claire : Oui, en fait ces connaissances ont un lien avec la militance, donc c'est vrai que... non, ce n'est pas que des homos parce qu'il y a des féministes, et ce sont des hétéros majoritairement.

- Enquêtrice: Les amis que vous côtoyez sont plutôt au départ des amis à toi ou à Hélène?

- Claire : C'est la question que l'on se posait une fois ; je ne sais pas. Maintenant on connaît tout le monde toutes les deux.

- Enquêtrice : Vos amis sont majoritairement homo, hétéro?

- Hélène : Les deux, je crois qu'on a quand même plus d'amis hétéro qu'homo mais les amis hyper proches, des copines bien proches sont homo... il y en a aussi qui sont proches et qui sont hétéro. Oui c'est un peu des deux. On est quand même bien entourées, si je compte tous les amis qu'on a, sans faire de nuance comme ça, on connaît plus d'hétéros que d'homos.

- Enquêtrice : C'est un espace plutôt ouvert?

- Hélène : Très ouvert.

Le deuxième ensemble regroupe cinq couples plutôt ouverts sur l'extérieur. On distingue, là encore, I'effectivité de deux réseaux relationnels distincts. Ils diffèrent toutefois par leurs étendues. La partenaire disposant du cercle amical le plus large encourage fréquemment les contacts que le couple entretient avec l'extérieur. Bien que partagé, le rôle de pourvoyeur de relations semble ainsi être davantage investi par l'une des deux partenaires. La comparaison de l'amplitude des réseaux de connaissances confirme cette observation. On relève une certaine concordance entre I'importance des cercles amicaux et de connaissances. La partenaire bénéficiant du réseau amical le plus fourni justifie également du réseau de connaissances le plus important. Ce constat semblerait s'expliquer par une promptitude plus marquée à investir des sphères extérieures au ménage, par un engagement dans des activités associatives ou sportives et l'exercice d'une activité professionnelle propice aux rencontres et aux sociabilités. Les réceptions sont fréquentes dans I'habitat mais se cantonnent à la sphère amicale, les connaissances n'étant que rarement conviées. Les entrées font ainsi l'objet d'une certaine sélection, I'habitat étant avant tout défini comme un espace d'intimité conjugale. Les réseaux relationnels sont empreints d'une mixité homosexuellehétérosexuelle. Cette mixité s'observe également dans les sociabilités au domicile. Toutefois, dans les discours, on note l'expression d'une familiarité et d'une complicité davantage marquées avec les amies lesbiennes. Cette proximité s'accompagne d'un mode de sociabilité spécifique. Alors que la venue des amis hétérosexuels relève souvent d'invitations formelles et programmées, celle des amies lesbiennes présente un caractère fréquent, régulier et improvisé. La spontanéité de ces visites amicales implique une intégration des convives dans I'habitat sans mise en scène préalable, soulignant ainsi une certaine aptitude à ouvrir son quotidien et son intimité conjugale. Chez ces couples, s'exprime une prépondérance de la conjugalité dans l'organisation des activités dans I'habitat. Le privilège accordé au temps et aux activités partagés se retrouve chez quatre couples, le cinquième se caractérisant par un équilibre entre les activités conjointes et les activités personnelles. La décoration n'est pas dépourvue d'une dimension conjugale; elle cristallise l'union chez trois couples. L'hégémonie du "nous» semblerait ne pas contrarier l'ouverture du couple sur l'extérieur, une ouverture globalement alimentée par les contacts qu'entretiennent les deux partenaires.

- Emma : On a beaucoup de potes. Je dirais que ce 
sont des potes aux deux mais peut-être un peu plus à Louise (...).

- Enquêtrice : Est-ce qu'il vous arrive d'inviter des connaissances chez vous?

- Emma : Rarement parce que c'est toujours le bordel. Il faut toujours ranger un maximum avant qu'il y ait quelqu'un qui vienne. Alors que les potes... tu as les vrais amis qui savent et qui s'en moquent un peu mais les potes ou les connaissances cela fait un peu mauvais effet, voilà c'est juste par rapport à ça (...). Tout ce qui est de la sphère professionnelle, personne ne vient. Les relations qu'on a ne justifient pas une invitation dans un cercle privé, je les vois au boulot et ça ne va pas plus loin. (...) C'est privé quand même ici, c'est pas un hall de gare chez moi [rire] (...). On invite le plus souvent des copines homo, des amies lesbiennes essentiellement, enfin je n'ai pas trop de souvenirs d'invitations hétéro. II n'y a pas d'occasion particulière, c'est quand ça nous chante de faire un truc; tu vois on prend le téléphone... Si on sait qu'il y a une fille qui est toute seule, on l'appelle pour savoir ce qu'elle fait pour qu'elle ne soit pas toute seule tout le temps.

- Louise : J'ai quelques connaissances. On n'en a pas en commun Emma et moi. J'aurais tendance à dire que j'en ai plus. Le problème c'est que, Emma, elle connaît beaucoup de monde mais elle ne les voit jamais. En termes de connaissances, de par les milieux qu'elle a fréquentés et les boulots qu'elle a fait, elle connaît beaucoup de monde mais on ne les voit jamais. Les gens qu'on est amenés à voir ponctuellement ce sont plus des gens qui sont en rapport avec moi, c'est lié à mes activités sportives. Pour les amis, c'est pareil, les amis qu'on voit essentiellement ce sont les miens, j'ai un certain nombre d'amis que je vois régulièrement. (...) La plupart de mes amies sont lesbiennes mais j'ai aussi des amis hétéro. Ce n'est pas le même type de relation. C'est vrai que j'ai peu d'amis proches hétéro mais, en même temps, ce n'est pas délibéré ni volontaire, c'est comme ça (...). Mes relations avec mes amies homo je dirais qu'elles sont plus profondes».

La troisième tendance regroupe trois couples faiblement ouverts à l'environnement. L'apport des contacts amicaux n'apparaît pas partagé et relève d'un unique réseau amical. L'aptitude à nourrir des échanges avec l'extérieur ne s'observe que chez l'une des deux conjointes et le rôle de pourvoyeur de relations n'est pas réparti. Le cercle amical du couple, appartenant initialement à une des conjointes, se compose essentiellement de couples lesbiens. La complicité et le vécu partagé de I'homosexualité semblent justifier ces liens électifs. Les échanges avec les amies lesbiennes sont fréquents mais programmés. Les visites impromptues sont dépréciées et les entrées dans l'habitat sont sélectives. Le réseau de connaissances n'est pas insignifiant mais est peu mobilisé. Les sociabilités dans I'habitat sont avant tout partagées avec les amies proches, les personnes de confiance. On entretient une certaine défiance à l'égard des personnes que l'on connaît peu, l'extérieur pouvant être jugé hostile. L'habitat se présente comme un espace à préserver d'autrui et fait l'objet $d^{\prime}$ 'une forte privatisation. Paradoxalement, I'organisation de ces trois couples ne concède pas une place de choix au "nous » conjugal. Elle semble plutôt exprimer un intérêt tantôt pour le couple, tantôt pour les individualités.

- Jeanne: Presque tous nos amis sont homos. Quand on sort, on sort avec eux, quand on va à l'association, il n'y a que des lesbiennes. Donc forcément, ça fait un peu ghetto dans le sens où de l'extérieur on pourrait croire qu'on ne veut pas voir d'autres gens alors que ce n'est pas le cas. Mais c'est juste la configuration qui est comme ça. Je pense qu'en fait Justine connaît beaucoup d'hétéros, mais dans un relationnel de connaissances plus que d'amis. Ce n'est pas un cercle amical proche, mais oui, elle en connaît plein des hétéros finalement, mais dans notre environnement proche, la plupart des gens sont homos.

- Enquêtrice: Est-ce que tu penses ton appartement comme un espace privé ?

- Jeanne : Ah oui, c'est chez moi. Il est privé parce que c'est notre endroit à nous, c'est là où on vit et tout le monde ne vient pas chez nous.

- Enquêtrice: Comment ça s'organise en fait quand tu dis que tout le monde ne vient pas chez vous?

- Jeanne: Moi je suis plutôt du genre à laisser entrer n'importe qui chez moi mais Justine un peu moins. Justine, il y a certaines personnes qu'elle ne veut pas inviter à la maison. Il faut vraiment qu'elle ait confiance en les personnes pour les laisser rentrer chez nous (...). Pour moi, ça n'engage pas tant que ça de laisser venir quelqu'un à la maison (...). Pour Justine ça engage peut-être plus de choses pour elle.

- Enquêtrice : Est-ce que tu as le sentiment que ton réseau de connaissances est important?

- Justine : Des personnes que je vois occasionnellement? non.

- Enquêtrice : Est-ce que tu as le sentiment d'avoir un nombre d'amis important?

- Justine: Non, de vrais amis non, je dirais pas important.

- Enquêtrice: Est-ce qu'il y a des gens que vous n'invitez jamais ?

- Justine : Ce sont des gens avec lesquels je ne suis pas à l'aise. Souvent pour moi rentrent à la maison les personnes en qui j'ai confiance et avec qui je me sens bien.

- Enquêtrice: Est ce que tu penses ton appartement comme un espace privé? 
- Justine : Oui.

- Enquêtrice : En quoi est-il privé ?

- Justine : Je décide qui rentre dans mon appartement. Je sais que je suis un peu... j'invite pas n'importe qui chez moi (...). Il y a des gens que je connais depuis des années et qui ne sont jamais venus chez moi et qui ne savent pas où j'habite. Oui, c'est un espace privé et c'est un espace de vie avec des témoignages de notre homosexualité, de notre vie de couple; donc, du coup, il y a des gens je n'ai pas envie qu'ils pénètrent là.

La dernière tendance réunit trois couples fermés sur l'extérieur. Chez ces couples, le cercle amical, fortement restreint, appartenait initialement à I'une des deux conjointes. Une même observation peut être rapportée pour le réseau de connaissances qui est, lui aussi, limité et peu mobilisé. Les contacts se limitent à un ou deux couples d'amis hétérosexuels et homosexuels ou se cantonnent à la famille. Les réceptions sont programmées et peu fréquentes. L'habitat est fortement associé à I'intimité conjugale et ne s'ouvre à ce titre qu'aux personnes les plus proches. II s'apparente, dans les discours, à «un nid», "un jardin » qui doit être préservé du regard extérieur. La stricte sélection des entrées permet de préserver l'équilibre et la stabilité de l'union. Deux de ces couples accordent un privilège aux activités communes dans I'habitat. Par ailleurs, on relève dans leurs décors intérieurs l'expression d'une individualité prépondérante. Associées à la clôture, ces deux caractéristiques confèrent à la relation un caractère fusionnel et laissent présager une répartition des tâches traditionnelle entre les conjointes.

- Enquêtrice: Qui est-ce que vous invitez le plus souvent chez vous?

- Carine: La famille, les sœurs, les parents (...). Pour la famille, il n'y a pas d'occasion, ça peut être un dimanche pour venir prendre un café, ça peut être un soir parce qu'ils sont à côté (...). Nous, avec notre famille, c'est toujours spontané.

- Enquêtrice : Et avec les amis?

- Carine : Toujours programmé [rire], c'est l'inverse jamais spontané (...). On programme une semaine avant pour le week-end d'après, déjà pour que tout le monde soit là et puis il faut qu'on s'organise avec les amis alors qu'avec la famille non (...).

- Enquêtrice: Quand tu dis que chez vous c'est aussi un lieu d'accueil, vous accueillez plutôt des proches?

- Carine: Oui ce sont des proches, il y a une sélection quand même (...).

- Enquêtrice : À l'inverse, est-ce qu'il y a des gens que vous n'invitez jamais?

- Carine : Oui, les connaissances, elles ne viennent pas parce que ce n'est pas des intimes. Ce qu'il y a à l'intérieur ça me reflète moi, la déco... C'est une partie de nous notre intérieur, donc les connaissances n'ont pas accès à ça (...). Ici, c'est la maison du bonheur et en dehors c'est la maison du cauchemar, mais au moins, là, c'est la maison du bonheur (...). Les autres, les hétéros, les gens, oui toute la civilisation, tous les gens, l'école primaire, les enfants, les maîtresses, tout le monde qui regarde, qui étiquette - parce qu'on est dans un pays qui étiquette - tous ces gens-là, c'est le monde extérieur. Je dirais que l'extérieur, c'est le cauchemar. On se cache chez nous, on est épanouies, heureuses, on fait ce qu'on veut, on dit ce qu'on veut.

- Enquêtrice : Est-ce que tu as le sentiment d'avoir un réseau de connaissances conséquent?

- Julie : Non, pas du tout même, non ça ne m'attire pas, connaître pour connaître non, je préfère approfondir.

- Enquêtrice : Et avant de te mettre en couple est-ce que tu avais un réseau de connaissances important? - Julie: Un peu plus oui (...). On peut avoir des coups de cour pour n'importe qui, et je pense $q u^{\prime}$ elle a plus peur de ça, qu'il y ait un danger à l'extérieur, que je puisse rencontrer quelqu'un ou que je tisse des liens amicaux qui lui déplaisent parce qu'elle est assez... pas envahissante mais elle aime bien être de partout, tout le temps avec moi. Elle aime bien voir tout ce que je peux faire, avec qui je suis, quand je rentre et tout (...). Nos amis, c'est son cercle à elle, elle me l'a un petit peu imposé entre guillemets parce que j'ai accepté donc ce n'est pas vraiment imposé.

- Enquêtrice : Est-ce qu'il vous arrive d'inviter des membres de votre famille?

- Julie : Oui, plus que les amis. Nos amis, on ne les invite pas souvent. Je dirais quand on peut. Il faut s'y prendre à l'avance pour passer des soirées avec eux (...).

- Enquêtrice : Est-ce qu'il y a des gens à qui tu n'ouvrirais pas la porte?

- Julie : Oui. À tout ce qui peut être de son côté, ses collègues, ses connaissances... ici, c'est mon jardin, c'est notre vie à nous, et ça, je ne le partage pas avec des gens que je ne connais pas trop, je ne veux pas qu'ils nous cernent.

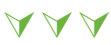

\section{Conclusion}

Le nombre de couples étudiés dans cet article est limité et n'offre, en ce sens, qu'un aperçu des organisations conjugales. Les conclusions ne prétendent ainsi qu'à une vision générale et pointent des pistes d'analyses à investiguer. En première observation, on relève des configurations conjugales disparates chez les couples lesbiens. Cette diversité rappelle celle qui contribue à qualifier les unions hétérosexuelles. Le couple, qu'il soit homosexuel ou hétérosexuel, semble 
des couples lesbiens enquêtés

\begin{tabular}{|c|c|c|c|c|c|}
\hline & \multirow[b]{2}{*}{ Décoration } & \multirow[b]{2}{*}{$\begin{array}{l}\text { Vie seule/ } \\
\text { vie avec }\end{array}$} & \multicolumn{2}{|c|}{ Ouverture/clôture du couple } & \multirow[b]{2}{*}{$\begin{array}{l}\text { Style de } \\
\text { conjugalité }\end{array}$} \\
\hline & & & $\begin{array}{l}\text { Rôle pourvoyeur } \\
\text { de relations et } \\
\text { entretien des } \\
\text { contacts avec } \\
\text { l'extérieur }\end{array}$ & Ouverture & \\
\hline $\begin{array}{l}\text { Agathe : chargée de clientèle groupe téléphonie ; } \\
\text { baccalauréat. } \\
\text { Émilie : serveuse dans la restauration ; niveau } \\
\text { seconde. }\end{array}$ & Nous & Nous & Les deux conjointes & Ouvert & Compagnonnage \\
\hline $\begin{array}{l}\text { Amandine : auxiliaire spécialisée vétérinaire ; } \\
\text { baccalauréat. } \\
\text { Charlotte : technicienne ; BTS Mécanique } \\
\text { Automatisme Industriel. }\end{array}$ & Nous & Je/Nous & Une conjointe & $\begin{array}{c}\text { Faiblement } \\
\text { ouvert }\end{array}$ & Bastion \\
\hline $\begin{array}{l}\text { Emma : vendeuse ; licence LEA. } \\
\text { Louise : professeur d'EPS ; CAPES. }\end{array}$ & Je & Nous & Une conjointe & Ouvert & $\begin{array}{l}\text { Bastion en tendance } \\
\text { car part des indivi- } \\
\text { dualités non négli- } \\
\text { geable }\end{array}$ \\
\hline $\begin{array}{l}\text { Julie : tapissière d'ameublement ; CAP tapissier. } \\
\text { Carine : gardienne de la paix ; Deug de droit. }\end{array}$ & Je & Nous & Une conjointe & Fermé & Cocon \\
\hline $\begin{array}{l}\text { Charline : comédienne humoriste ; } \\
\text { BEP sténodactylo. } \\
\text { Lucie : professeur de français ; CAPES. }\end{array}$ & Je & Je/Nous & Une conjointe & Ouvert & $\begin{array}{l}\text { Bastion en tendance } \\
\text { car part des indivi- } \\
\text { dualités non négli- } \\
\text { geable }\end{array}$ \\
\hline $\begin{array}{l}\text { Claire : médecin généraliste ; doctorat de } \\
\text { médecine. } \\
\text { Hélène : assistante sociale ; master sciences politiques. }\end{array}$ & $\mathrm{Je} / \mathrm{Je}$ & Je/Nous & Les deux conjointes & Très ouvert & Association \\
\hline $\begin{array}{l}\text { Cécile : chef de cuisine ; BEP cuisine. } \\
\text { Natacha : conditionneuse ; aucun diplôme. }\end{array}$ & $\mathrm{Je} / \mathrm{Je}$ & Je/Nous & Une conjointe & Fermé & $\begin{array}{l}\text { Cocon en tendance, } \\
\text { emprunte également } \\
\text { au style "parallèle » }\end{array}$ \\
\hline $\begin{array}{l}\text { Raphaëlle : chômage ; BEP agricole. } \\
\text { Delphine : éducatrice jeunes enfants ; diplôme } \\
\text { d'État d'éducatrice. }\end{array}$ & $\mathrm{Je} / \mathrm{Je}$ & Je/Nous & Les deux conjointes & Très ouvert & Association \\
\hline $\begin{array}{l}\text { Claudine : journaliste ; Maîtrise d'études } \\
\text { théâtrales. } \\
\text { Stéphanie : patronne d'un bar ; } \\
\text { CAP prothésiste dentaire. }\end{array}$ & $\begin{array}{l}\text { ni Je } \\
\text { ni Nous }\end{array}$ & $\mathrm{Je} / \mathrm{Je}$ & Les deux conjointes & Très ouvert & Association \\
\hline $\begin{array}{l}\text { Cécile : employée grande distribution ; aucun } \\
\text { diplôme. } \\
\text { Amandine : adjoint administratif dans une } \\
\text { université ; maîtrise de lettres modernes. }\end{array}$ & $\begin{array}{l}\text { ni Je } \\
\text { ni Nous }\end{array}$ & $\mathrm{Je} / \mathrm{Je}$ & Une conjointe & $\begin{array}{c}\text { Faiblement } \\
\text { ouvert }\end{array}$ & Parallèle \\
\hline
\end{tabular}

Source : ces résultats d'analyse sont issus de la recherche doctorale en cours, Costechareire C., "Les parcours homosexuels et les styles de conjugalité chez les lesbiennes » (titre provisoire), université Lumière Lyon 2 - Centre Max Weber.

Nous : hégémonie du "nous » conjugal ; Je : hégémonie d’une individualité ; Je/Je : hégémonie des deux individualités ; ni Je ni nous : pas d’hégémonie; Je/Nous : équilibre entre les individualités et le couple. 
toujours s'inscrire dans un jeu de négociations interpersonnelles dont les règles fluctuent au gré du dialogue qui s'établit entre le «je» et le " nous ", et au gré de la propension à être ouvert ou fermé à l'environnement. On ne saurait sur ce point accorder aux unions féminines une quelconque particularité. À l'échelle de l'échantillon étudié, il ne ressort pas de style conjugal prépondérant. La diversité implique des modes d'organisation tantôt orchestrés par la fusion et/ou une répartition des tâches marquée entre les conjointes (styles bastion, cocon et parallèle), tantôt ouverts aux échanges et à l'autonomie dans le couple (styles association et compagnonnage). Les disparités qui s'illustrent dans ces différentes " manières de faire couple» sembleraient s'éclairer à la lumière de différents facteurs. Certaines renvoient au poids de la norme et de la conformité. L'adhésion à un modèle conjugal traditionnel sous-tend, dans ces quelques cas, l'influence de I'hétéronormativité qui s'exprime alors par une répartition stricte des rôles et des tâches entre les partenaires. Bien qu'elle n'orchestre pas la globalité des tâches et des rôles au sein du couple, la dimension genrée de la répartition apparaît heuristique. Elle s'associe, en outre, à une conformité au modèle hétérosexuel qui s'exprime de manière prégnante dans les pratiques et dans les discours. Ce cas de figure ne fait toutefois pas I'unanimité.
À l'instar des couples hétérosexuels, les couples lesbiens répondent, par les négociations inhérentes à leur relation, aux nouvelles exigences conjugales. Ces couples trouvent ainsi leur "mesure » au gré des ajustements entre les partenaires et d'une adhésion plus ou moins forte à un modèle conjugal préexistant. Le poids des socialisations antérieures et des savoir-faire de chaque conjointe apparaît non négligeable mais ne saurait expliquer les configurations conjugales à lui seul. En effet, le style de la relation s'élabore également par les interactions, par les capacités et la volonté des protagonistes à concéder ou à accepter certains rôles. L'étude des styles de conjugalité chez les couples lesbiens invite ainsi à poursuivre l'analyse des logiques régissant les échanges et les consensus dans la relation. Elle questionne, en outre, les catégories et la pertinence d'un mode de pensée dichotomique fondé sur la distinction des couples hétérosexuels et des couples homosexuels. La pluralité des styles de conjugalité qui s'observe de part et d'autre suggère de penser le couple quelle que soit la sexualité. Aussi, à l'instar de ce que propose Irène Théry: "(...) il est sans doute temps de poser le principe d'incertitude le plus simple et le plus évident : il n'y a qu'une sexualité humaine (...). De même que le droit ne s'occupe pas de l'hétérosexualité mais du couple ou de la filiation, c'est bien le couple de même sexe et la filiation que le droit doit désormais prendre en compte » (Théry, 2005:171).

\section{Références bibliographiques}

- Allen B., 2004, Les relations entre le dedans et le dehors, in Espaces domestiques (sous la dir. de Collignon B. et Staszak J.-F.), Bréal.

- Authier J.-Y., 2001, «Espace et socialisation. Regards sociologiques sur les dimensions spatiales de la vie sociale ", université Lumière Lyon 2/faculté d'anthropologie et de sociologie.

- Bernard Y., 1998, Du logement au chez-soi, in Logement et habitat, l'état des savoirs (sous la dir. de Segaud M., Bonvalet C. et. Brun J.), Liège, La Découverte:374-381.

- Bonnin P., 1990, Produire la domus : une affaire de famille, Niveaux et formes d'investissement des familles dans l'espace domestique, Sociétés contemporaines, $n^{\circ}$ 5:145-158.

- Bonvalet C. et Clément C., 2005, Familles recomposées et ancrage résidentiel, Espaces et Sociétés, $\mathrm{n}^{\circ}$ 120-121:79-97.

- Chetcuti N., 2010, Se dire lesbienne. Vie de couple, sexualité et représentations de soi, Paris, Payot.

- Clair I., 2007, Dire à deux le ménage, in L'injustice ménagère [sous la dir. de Singly F. (de)], Paris, Armand Colin:179-222.

- Costechareire C., 2008, Les "parcours homosexuels » et conjugaux au sein d'une population lesbienne, Enfances, Familles, Générations, $n^{\circ}$ 9, URL: http://www.erudit.org/revue/efg/2008/v/n9/index.html

- Courduriès J., 2008, "La conjugalité des couples gays en France dans les années 2000 », thèse d'anthropologie sous la dir. d'Agnès Fine, université de Toulouse-Le Mirail.

- Courduriès J., 2006, Les couples gays et la norme d'égalité conjugale, Ethnologie française, tome XXXVI:705-711.

- Déchaux J.-H., 2007, Sociologie de la famille, Paris, La Découverte.

- Descoutures V., 2010, Les mères lesbiennes, Paris, PUF. 
- Descoutures V., 2008, Les mères lesbiennes et la figure du garant, in Mariages et homosexualités dans le monde. L'arrangement des normes familiales (sous la dir. de Descoutures V., Digoix M., Fassin É. et Rault W.), Paris, Éditions Autrement:165-176.

- Eleb M., 2004, Le roman du couple et sa scène spatiale, in Espaces domestiques (sous la dir. de Collignon B. et Staszak J.-F.), Paris, Bréal:310-324.

- Faure-Rouesnel L., 2006, Cacher, se montrer : le chez-soi et le choix de la liste de mariage à Londres, in (sous la dir. de Gherchanoc F.), La maison, lieu de sociabilité, dans des communautés urbaines, européennes, de l'Antiquité à nos jours, Paris, Éditions Le Manuscrit:207-233.

- Faure-Rouesnel L., 2004, Nos débuts ensemble, installation résidentielle et entrée dans la conjugalité, Espaces domestiques (sous la dir. de Collignon B. et Staszak J.-F.), Paris, Bréal:325-340.

- Ferzli R., 2001, Couples au féminin : aspects du quotidien, Cahiers du Genre, n 30:147-178.

- Gherchanoc F., 2006, La maison à l'intersection du privé et du public : la sociabilité en question, in La maison, lieu de sociabilité, dans des communautés urbaines, européennes, de l'Antiquité à nos jours (sous la dir. de Gherchanoc F.), Paris, Éditions Le Manuscrit:11-34.

- Goldberg A. et Allen K., 2009, La perception de la participation masculine pour les mères lesbiennes lors du passage à la parentalité, Politiques sociales et familiales, $n^{\circ}$ 96:55-70.

- Grafmeyer Y., 1995, Sociologie urbaine, Lassay-les-Châteaux, Nathan.

- Gratton E., 2008, L'homoparentalité au masculin. Le désir d'enfant contre l'ordre social, Paris, PUF.

- Gross M., 2009, Les familles homoparentales : entre conformité et innovations, Informations sociales, $\mathrm{n}^{\circ} 154: 106-114$

- Gross M., 2005, Homoparentalités, état des lieux, Ramonville St-Agne, Érès.

- Kaufmann J.-C., 2003, Sociologie du couple, Paris, PUF, collection Que sais-je ?

- Kaufmann J.-C., 1992, La trame conjugale. Analyse du couple par son linge, Paris, Nathan, collection Pocket.

- Kaufmann J.-C., 1991, Les habitudes domestiques, in F. de Singly (dir), La famille-l'état des savoirs [sous la dir. de Singly F. (de)], Paris, La Découverte:124-132.

- Kellerhals J., Widmer E., Levy R., 2004, Mesure et démesure du couple. Cohésion, crises et résilience dans la vie des couples, Paris, Payot ( $2^{\mathrm{e}}$ édition en 2008 dans la collection Petite bibliothèque Payot).

- Lawrence R. J., 1982, L'espace domestique : typologie et vécu, Cahiers internationaux de sociologie, vol. LXXII, Seuil:67-75.

- Le Gall D., 2005, La conception de l'habiter à l'épreuve de la recomposition familiale, Espaces et Sociétés, $\mathrm{n}^{\circ}$ 120-121:45-60.

- Lerch A., 2007, Normes amoureuses et pratiques relationnelles dans les couples gays. Héritage et inventivité ?, Informations sociales, $\mathrm{n}^{\circ}$ 144:108-117.

- Mailfert M., 2007, L'entourage social des familles homoparentales : une gestion différentielle du comment se dire ou se faire couple parental quand on forme un couple homosexuel, in Le choix de I'homosexualité (sous la dir. de Perreau B., Handman M.-E. et Gaspard F), Paris, EPEL:209-228.

- Mailfert M., 2005, La famille homoparentale au féminin : constitution, fonctionnement et processus de légitimation, L'esprit du temps, $\mathrm{n}^{\circ}$ 38:171-185.

- Mallon I., 2004, Le chez-soi des personnes âgées en maison de retraite, in Espaces domestiques (sous la dir. de Collignon B. et Staszak J.-F.), Paris, Bréal:370-382.

- Poittevin A., 2005, Une lecture des espaces pour lire les liens fraternels, Espaces et Sociétés, n 120-121:61-77.

- Prost A., 1999, Frontières et espaces du privé, in Histoire de la vie privée. De la Première Guerre mondiale à nos jours (sous la dir. de Ariès P. et Duby G.), tome V, Manchecourt, Seuil:13-97.

- Rissoan O., 2004, Les sociabilités amicales des jeunes : un analyseur des trajectoires sociales lors du passage à l'âge adulte, Genèses, n 54:148-161.

- Segalen M. et Le Wita B., 1993, Éditorial, in Chez-soi. Objets et décors : des créations familiales ? (sous la dir. de Segalen M. et Le Wita B.), Paris, Éditions Autrement, n 137:11-23.

- Singly F. (de), 2008, Les uns avec les autres. Quand l'individualisme crée du lien, Paris, Hachette Littératures.

- Singly F. (de), 1996, " Habitat et relations familiales », rapport pour le Plan Construction et Architecture (ministère du Logement), université de Paris-5.

- Théry I., 2005, Différence des sexes, homosexualité et filiation, in Homoparentalités, états des lieux (sous la dir. de Gross M.), Paris, Érès:151-178. 
